\title{
Brain Mri Image Segmentation Using Simple Linear Iterative Clustering (SLIC) Segmentation With Superpixel Fusion
}

\author{
Snehalatha and Siddarama R Patil \\ Department, Poojya Doddappa Appa College of Engineering, (Affiliated to \\ Visvesvaraya Technological University) Kalaburagi, India
}

\begin{abstract}
Brain MRI image segmentation is a challenging task owing to the complex texture of the various parts of the brain. The goal of the segmentation is to divide the image into parts so that the classification algorithm can recognize the tumour present in the image. This paper presents a modified Simple Linear Iterative Clustering (SLIC) segmentation algorithm to segment the brain MRI image. The SLIC segmentation divides the brain MRI image into many superpixels. This algorithm uses both spatial intensity value and the pixel positions to form the superpixels. These superpixels are then fused with the neighbouring superpixels to form bigger regions in order to merge similar regions. The fusion operation is implemented to merge the superpixels which constitute the tumour in the image. This would help the classification algorithms to perform better. The proposed segmentation method is compared with existing techniques to analyse the performance. The existing algorithms are compared in terms of execution time, average deviation from and entropy. The experimental results prove that the proposed method segmented the brain MRI image better than the existing algorithms.
\end{abstract}

\section{KEY WORDS: BRAIN MRI, SEGMENTATION, TUMOUR, SIMPLE LINEAR ITERATIVE CLUSTERING (SLIC).}

\section{INTRODUCTION}

In the body, the most important part is brain which has included the most complex structure. The study of operations and the increment of diagnosis of diseases complexity are hindered by the presence of skull around the brain. Any specific diseases couldn't be liable to the brain like the other remaining parts of the body. But, the growth of cells abnormally can be triggered where a change in its structure and behaviour is made possible. This abnormality feature is indicated the Brain Tumor usually. To detect such type of tumors, MRI is utilized. By including the technology improvements, a researched area has considered the brain tumor and tissue segmentation.

For discriminating and predicting the better points of interest in the body structure, MRI is used specifically on behalf of a biomedical system. The differences in the

Biosc Biotech Res Comm P-ISSN: 0974-6455 E-ISSN: 2321-4007
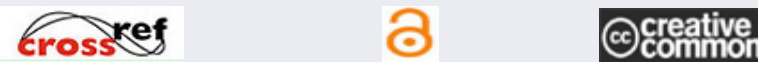

Identifiers and Pagination

Article Information

Year: 2021 Vol: 14 No (5) Special Issue

Received: $19^{\text {th }}$ Mar 2021

Pages: 358-364 Accepted after revision: $30^{\text {th }}$ May 2021

This is an open access article under Creative

Commons License Attribn 4.0 Intl (CC-BY).

DOI: http://dx.doi.org/10.21786/bbrc/14.5/62 tissues that have a much better technique in contrary to the estimated tomography can be distinguished by exploiting this system fundamentally. To identify the brain tumor and growth imaging, this procedure is made an extraordinary and exceptional type. As a part of various examinations, MRI is exploited widely in the medical field. For making the image of a body, MRI techniques are used as radio waves and powerful magnet as it is a non-invasive method. To probe the soft tissues of the human body, this technique is suitable. For example, brain tumors, spinal cord injury, Ligament and tendon damage, etc. Several MRI image segmentations have been proposed by researcher. Some of such categories are clustering based techniques, region growing and merging and semantic segmentation. This paper presents a SLIC super pixel segmentation with superpixel fusion. The input data is first given to the SLIC algorithm. Superpixel fusion takes place on the resultant superpixels with similar textural features.

Proposed Method: In the proposed method, the brain MRI image is given to the SLIC superpixel algorithm first. The formed superpixels are then fused with the neighbouring superpixels which possess similar structure. Figure 1 . Shows the block diagram representation of the proposed model. The implementation of the two modules, namely SLIC superpixel segmentation and fusion of superpixels are presented in the following subsections.

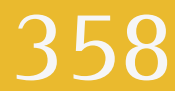


Figure 1: Block diagram of proposed model

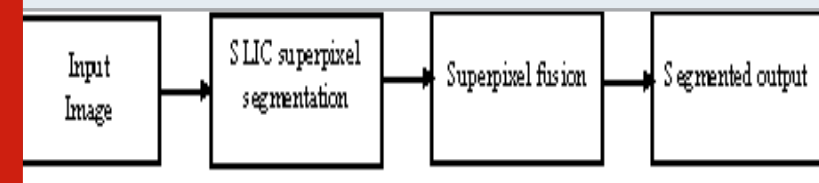

SLIC segmentation: The SLIC algorithm generates super pixels with a simple iterative algorithm. The use of super pixels instead of pixels offers on the one hand the advantage that the computing time is significantly reduced during classification. In addition, the super pixels have an additional smoothing effect. Misclassification of isolated pixels are avoided because all pixels assigned within a superpixel have the class. Two nodes are considered to be adjacent within a level if the edge polygons of the associated image have a common edge. The statistical dependencies between different parts of the tumour effect the segmentation accuracy.

Figure 2: Sketch of the search area around a cluster centre. $S$ is the distance between the cluster centres.

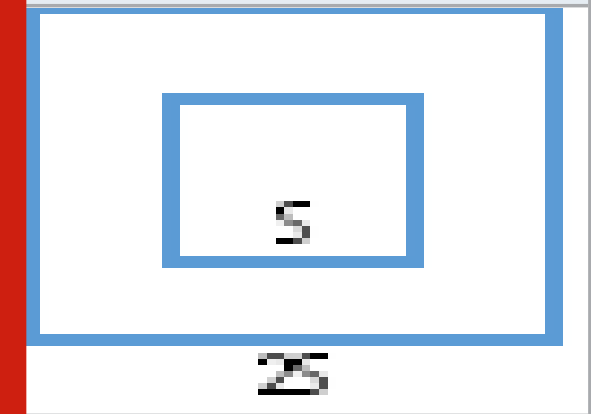

Let $\mathrm{k}$ be the number of cluster centres in an image. The size of each superpixel is calculated from $\mathrm{k}$ and the number of pixels $\mathrm{N}$ of the image. In the first step, $\mathrm{k}$ cluster centres are regularly distributed over the image. This happens at a distance $=\mathrm{N} / \mathrm{k}$. Each cluster receives a search area with the size $2 \mathrm{Sx} 2 \mathrm{~S}$, which is shown in Fig 2. This limitation of the search area means that the number of required distance calculations are severely limited and the complexity of the algorithm is linear. The distance calculation for each pixel, the distances to the cluster centres are calculated, in whose search area range the pixel lies. The pixel then becomes the cluster with the closest spacing assigned.

For the distance calculation, a 5-dimensional vector is used for each pixel $|\mathrm{dl} d \mathrm{da} \mathrm{db} \mathrm{dx} \mathrm{dy}|^{\mathrm{T}}$ set up. The vector|dl $\left.\mathrm{da} d \mathrm{db}\right|^{\mathrm{T}}$ stands for the pixel color in the CIELAB colour space. This three-dimensional colour space divides colours based on their hue and brightness ability (blackand-white scale). In this way, colour differences can be described as distance. The vector $\mid \mathrm{dx}$ dy|represents the spatial distance on the picture. The distance between the colour values is limited to the size of the CIELAB colour space and the spatial distance in the picture to the picture size. Therefore the spatial distance must be normalized, so that both distances have the same influence on the result.

$$
\begin{aligned}
& d_{l a b}=\sqrt{\left(d l_{k}-d l_{i}\right)^{2}+\left(d a_{k}-d a_{i}\right)^{2}+\left(d b_{k}-d b_{i}\right)^{2}} \\
& d_{x y}=\sqrt{\left(d x_{k}-d x_{i}\right)^{2}+\left(d y_{k}-d y_{i}\right)^{2}} \\
& D_{s}=d_{l a b}+\frac{m}{s} d_{x y}
\end{aligned}
$$

Where,

$d_{l a b}$ is the distance between intensity values.

$d_{x y}$ is the spatial pixel distance.

$m$ is compactness.

$s$ is cluster distance.

$D_{S}$ is the spatial distance being normalized with the aid of the cluster distance $s$.

In the next step, the cluster centres of all super pixels are recalculated. To do this, the mean value for each entry of the distance vector is formed from all the pixels in the cluster. The distances to all cluster centres in whose search areas the respective pixel is calculated and the algorithm will run again. Figure 3 represents the Brain tumour MRI image and superpixel segmentation image. This is done iteratively until the cluster centres barely move. Overall, the SLIC algorithm requires the following input parameters:

- Color Channel 1-3: Here three primary channels R, $\mathrm{G}$ and $\mathrm{B}$ that will later be transferred to the CIELAB Color space which have an influence on the limits of the superpixels.

- Minimum and maximum size of the superpixels: Minimum and maximum size that the superpixels should have.

- Compactness $m$ of the super pixels: This parameter indicates how compact the individual pixels should be. The larger the value for $\mathrm{m}$, the more influence on the spatial distance and has the less the super pixels adapt to color jumps in the image.

\begin{tabular}{|ll|}
\hline \multicolumn{1}{|c|}{ Algorithm 1: SLIC superpixel algorithm } \\
\hline 1. & Initialize the cluster centres $C_{k}=|d l d a d b d x d y|^{T}$ \\
2. & For every cluster centre $C_{k}$, \\
3. & Find the pixels similar to the cluster centre in the neighbourhood of $2 S \times 2 S$ using the eq. (3). \\
4. & If the pixel is similar to the cluster centre, group it with the same cluster centre. \\
5. & If the $D_{s}$ value is more, merge it with the cluster with the least $D_{s}$. \\
6. & Repeat the steps 2 to 5 till the entire image is segmented. \\
\hline
\end{tabular}

Figure 3: (a) Brain tumour MRI image and (b) superpixel segmentation image

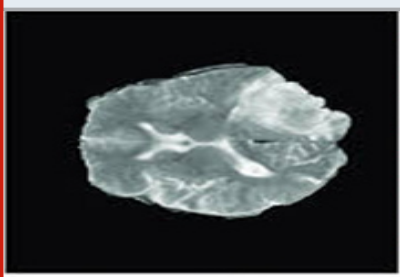

(a)

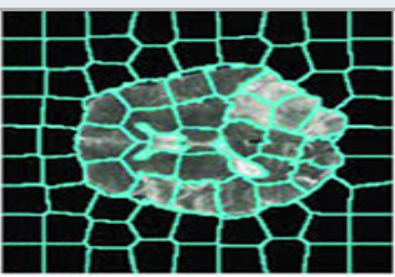

(b) 
2.2 Fusion of super pixels: Density-related fusion methods recognize areas with similar characteristics as clusters. First, the density of a particular superpixel has to be measured. In the case of the center-based approach, the density for the superpixel $p$ is estimated by the number of superpixels in an $\varepsilon$ region. The $\varepsilon$ - neighborhood of $p$ consists of all superpixels which are at most the distance $\varepsilon$ from $p$.

If the radius $\varepsilon$ is very large, then all data superpixels have the density $\mathrm{m}$ (= number of data superpixels), since then for each superpixel $p$ all superpixels lie in the $\varepsilon$ - neighborhood of p. If $\varepsilon$ is chosen too small, each superpixel has the density 1 . Therefore, the determination of $\varepsilon$ in the run-up to the analysis is of central importance. Another parameter that is considered is MinPixCnt. This specifies how many superpixels must lie in the $\varepsilon$ neighborhood of a superpixel $p$ so that $p$ belongs to a cluster.

The segmented image superpixels are divided into three different categories. A data superpixel lies either inside a dense region (core superpixel), on the edge of such a region (edge superpixel) or in a sparsely occupied area (noise superpixel) more precisely:

- Core superpixel:The number of data superpixels in the $\varepsilon$ neighbourhood of the core superpixel is at least MinPixCnt.

- Edge superpixel:An edge superpixel is not a core superpixel, but lies in the $\varepsilon$ - neighbourhood of a core superpixel.

- Intoxication superpixel:An intoxication superpixel is neither a core nor an edge superpixel.

- Informally described, two core superpixels whose distance is at most $\varepsilon$ land in the same cluster, edge superpixels are assigned to the cluster of a corresponding core superpixel and noise superpixel are neglected.

\begin{tabular}{|c|}
\hline Algorithm 2: Superpixixel fusion \\
\hline 1. Name the superpixixels as core, edge, or noise superpixixls. \\
\hline 2. Delete all intoxication superpixels. \\
\hline 3. Commect core superpixels that lie within an $\&$-ball with an edge. \\
\hline 4. Assign each edge superpixal to the cluster of an adijacent core superpixel. \\
\hline
\end{tabular}

In order for superpixel fusion to provide good clustering, the values of the parameters $\varepsilon$ and MinPixCnt must be chosen carefully. The usual approach to determining these values is to consider the distance between a superpixel $p$ and its $\mathrm{k}$-nearest neighbor, i.e., the superpixel that has the $\mathrm{k}$ distance from $\mathrm{p}$. For superpixels within a cluster, $\mathrm{k}$ - dist is usually small,

Experimental Results: The proposed method is tested on different types of MRI images described here:
- Susceptibility weighted imaging (SWI): SWIis a recently popular MRI technique primarily used when neurologic disorders needs to be diagnosed. The MRI highlights the differences in the magnetic susceptibility of the brain components like blood, iron, deoxygenated blood and calcium, thereby increasing the contrast in the MRI.

- Apparent diffusion coefficient (ADC): $A D C$ is an MRI image which is a modified version of a DWI without the $\mathrm{T} 2$ component.

- T1 weighted:T1 weighted image is the most commonly used MRI image sequence. The image clearly depicts the appearances of tissues macroscopically.

- T2 weighted: The T2 weighted image is also a very widely used MRI image which retains the dominant signal intensities. The MRI depicts fluid, muscle, fat, grey and white matter.

- DWI: The DWI image is an isotropic T2 weighted map which is formed by the T2 signal and the diffusion values. The MRI image presents the grey matter, white matter, CSF, Fat and other soft tissues.

- Fluid attenuated inversion recovery (FLAIR): FLAIR MRI removed the signal form the cerebrospinal fluid thereby resulting in a special inversion recovery sequence.

- T2*weighted: $2^{*}$ imaging is an MRI sequence to quantify observable or effective T2 (T2* or "T2-star"). In this sequence, haemorrhages and hemosiderin deposits become hypo intense.

- PD weighted: Given that nuclear magnetic resonance of protons (hydrogen ions) forms the major basis of MRI, it is not surprising that signal can be weighted to reflect the actual density of protons; an intermediate sequence sharing some features of both $\mathrm{T} 1$ and T2. The components depicted in the MRI include fluid, muscle, fat, hyaline cartilage and fibrocartilage. The following table 1 shows the segmentation results of the proposed method on different MRI sequences stated above.

The proposed method is compared with existing techniques k-means and mean shift segmentation in terms of elapsed time, mean value, average deviation from mean and entropy.

Elapsed time: Time taken by the algorithm to segment the image.

Mean value: The mean pixel value of the segment.

Average Deviation: This value represents the deviation of the pixels values in a cluster from its mean. Lesser the deviation, better the segmentation.

Entropy: Entropy measures the randomness of the pixel values present in the segment. Lesser the entropy, better the segmentation by an algorithm. 
In the tables 2 to 5, the elapsed time, mean, average deviation from mean and the entropy are all observed as minimum in the proposed method. This proves that the proposed method has performed better when compared to the exiting segmentation algorithms.

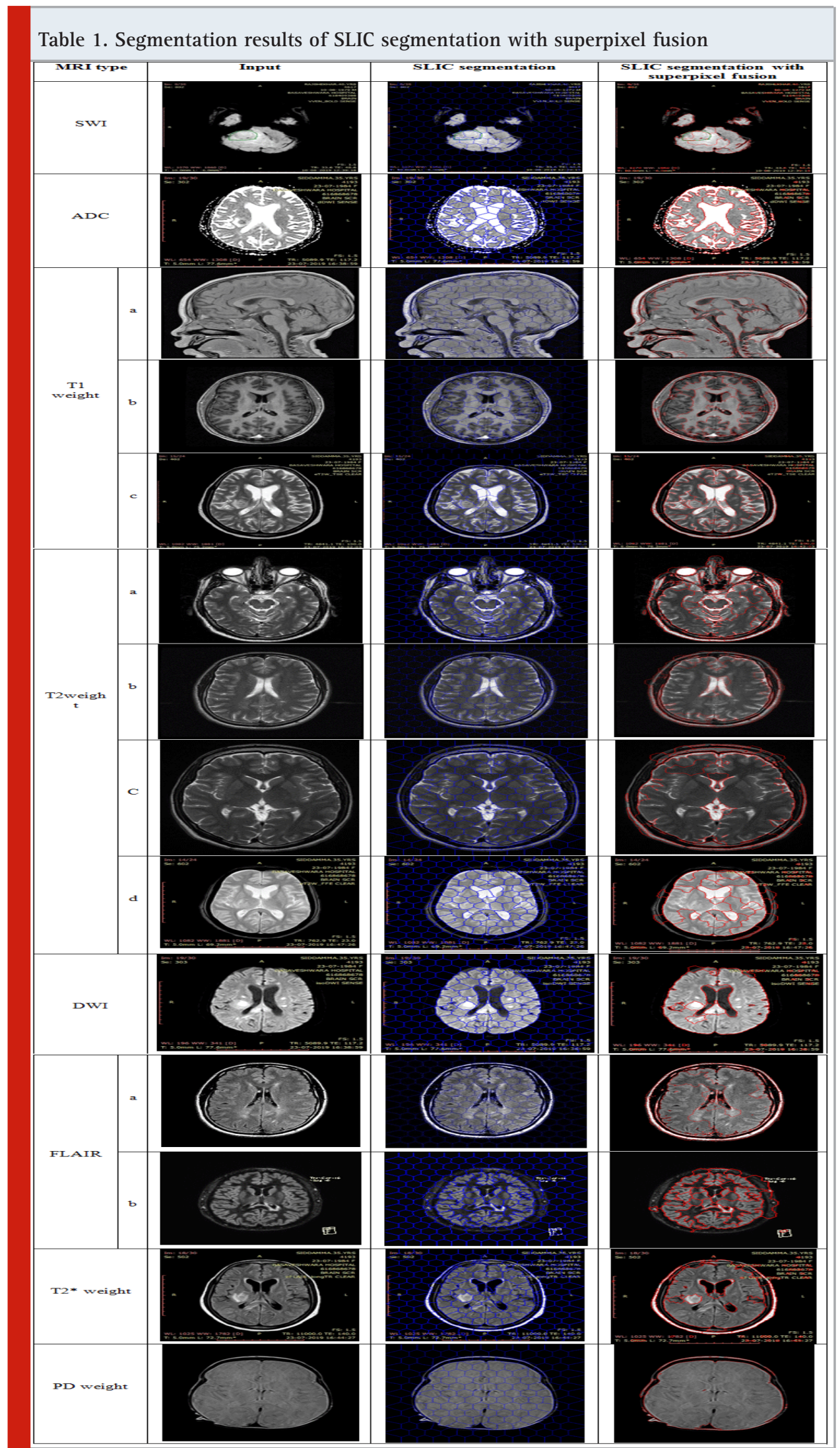


Table 2. Elapsed time

\begin{tabular}{|l|c|c|c|}
\hline Image & K-means [16] & Mean shift [17] & Proposed algorithm \\
\hline adc & 2.238980 & 0.714511 & 0.078972 \\
\hline conv dw1 & 2.734809 & 0.647594 & 0.038209 \\
\hline $\begin{array}{l}\text { multiple-sclerosis-35 } \\
\text { mripediatric brain pd axial }\end{array}$ & 1.522309 & 0.071121 & 0.037827 \\
\hline image 3 & 10.782455 & 0.791066 & 0.158915 \\
\hline sus wat & 10.769653 & 6.676479 & 0.190005 \\
\hline $\begin{array}{l}\text { t1 } \\
\text { brain_mri_ }\end{array}$ & 9.208027 & 4.213025 & 0.145144 \\
\hline transversal_t2_002 & 4.458631 & 0.981096 & 0.109113 \\
\hline t2(star) & 2.419330 & 0.649109 & 0.042103 \\
\hline
\end{tabular}

Table 3. Mean value

\begin{tabular}{|l|c|c|c|}
\hline Image & K-means [16] & Mean shift [17] & Proposed algorithm \\
\hline adc & 151.136835 & 141.430345 & 129.321684 \\
\hline conv dw1 & 100.040041 & 136.072562 & 64.179059 \\
\hline $\begin{array}{l}\text { multiple-sclerosis-35 } \\
\text { mripediatric brain pd axial }\end{array}$ & 76.571196 & 121.491920 & 43.597124 \\
\hline image 3 & 83.442380 & 97.833810 & 71.764321 \\
\hline sus wat & 80.566957 & 137.691116 & 69.965320 \\
\hline $\begin{array}{l}\text { t1 } \\
\text { brain_mri_ }\end{array}$ & 107.174528 & 143.401308 & 105.321873 \\
\hline transversal_t2_002 & 76.429280 & 136.600206 & 63.440623 \\
\hline t2(star) & 82.341560 & 130.319726 & 57.921296 \\
\hline
\end{tabular}

Table 4. Average Deviation from Mean

\begin{tabular}{|l|c|c|c|}
\hline Image & K-means [16] & Mean shift [17] & Proposed algorithm \\
\hline adc & 35877.410923 & 17290.578828, & 26997.993388 \\
\hline conv dw1 & 33298.972565 & 13610.956284 & 19017.557552 \\
\hline $\begin{array}{l}\text { multiple-sclerosis-35 } \\
\text { mripediatric brain pd axial }\end{array}$ & 1447.251152 & 3303.840799 & 9116.401200 \\
\hline image 3 & 35744.226387 & 729967.238157 & 25188.935943 \\
\hline sus wat & 75063.640345 & 21717.469726 & 47222.462397 \\
\hline $\begin{array}{l}\text { t1 } \\
\text { brain_mri_ }\end{array}$ & 103673.064028 & 33373.045462 & 80759.569904 \\
\hline transversal_t2_002 & 81015.446730 & 90530.931883 & 37284.545856 \\
\hline t2(star) & 25938.206637 & 1696.789390 & 22474.284669 \\
\hline
\end{tabular}




\begin{tabular}{|c|c|c|c|}
\hline Image & K-means [16] & Mean shift [17] & $\begin{array}{c}\text { Superpixel } \\
\text { segmentation }\end{array}$ \\
\hline adc & 35877.410923 & 17290.578828, & 26997.993388 \\
\hline $\begin{array}{l}\text { conv dw1 } \\
\text { multiple-sclerosis-35 }\end{array}$ & $\begin{array}{c}33298.972565 \\
1447.251152\end{array}$ & $\begin{array}{c}13610.956284 \\
3303.840799\end{array}$ & $\begin{array}{c}19017.557552 \\
9116.401200\end{array}$ \\
\hline \multicolumn{4}{|c|}{ mripediatric brain pd axial } \\
\hline image 3 & 35744.226387 & 729967.238157 & 25188.935943 \\
\hline $\begin{array}{l}\text { sus wat } \\
\text { t1 }\end{array}$ & $\begin{array}{c}75063.640345 \\
103673.064028\end{array}$ & $\begin{array}{l}21717.469726 \\
33373.045462\end{array}$ & $\begin{array}{l}47222.462397 \\
80759.569904\end{array}$ \\
\hline \multicolumn{4}{|l|}{ brain_mri_ } \\
\hline transversal_t2_002 & 81015.446730 & 90530.931883 & 37284.545856 \\
\hline t2(star) & 25938.206637 & 1696.789390 & 22474.284669 \\
\hline
\end{tabular}

\section{CONCLUSION}

This paper presents a SLIC superpixel segmentation algorithm with superpixel fusion from brain MRI image segmentation. SLIC superpixel segmentation is generally used for satellite image segmentation where the images are very complex. As the brain MRI image has complex textures, the SLIC segmentation algorithm can provide better results when compared to the existing techniques. The proposed method used SLIC superpixel segmentation along with superpixel fusion which combines similar superpixels facilitating the classification process. The proposed algorithm is implemented over different kinds of MRI images namely, Susceptibility weighted imaging (SWI), Apparent diffusion coefficient (ADC), T1 weighted, T2 weighted, DWI, Fluid attenuated inversion recovery (FLAIR), T2* weighted and PD weighted. The segmentation algorithm performed better on all types of MRI image. The minimum values of elapsed time, mean, average deviation from mean and the entropy prove that the proposed method performed a better segmentation of the image.

\section{REFERENCES}

Al-Kafri, Ala S., Sud Sudirman, Abir Hussain, Dhiya Al-Jumeily, Friska Natalia, Hira Meidia, Nunik Afriliana, Wasfi Al-Rashdan, Mohammad Bashtawi, and Mohammed Al-Jumaily. "Boundary delineation of MRI images for lumbar spinal stenosis detection through semantic segmentation using deep neural networks." IEEE Access 7 (2019): 43487-43501.

Arunkumar, N., Mazin Abed Mohammed, Mohd Khanapi Abd Ghani, Dheyaa Ahmed Ibrahim, Enas Abdulhay, Gustavo Ramirez-Gonzalez, and Victor Hugo C. de Albuquerque. "K-means clustering and neural network for object detecting and identifying abnormality of brain tumor." Soft Computing 23, no. 19 (2019): 90839096.

Bernard, Olivier, Alain Lalande, Clement Zotti, Frederick
Cervenansky, Xin Yang, Pheng-Ann Heng, Irem Cetin et al. "Deep learning techniques for automatic MRI cardiac multi-structures segmentation and diagnosis: is the problem solved?." IEEE transactions on medical imaging 37, no. 11 (2018): 2514-2525.

Despotovi刃, Ivana, Bart Goossens, and Wilfried Philips. "MRI segmentation of the human brain: challenges, methods, and applications." Computational and mathematical methods in medicine 2015 (2015).

Gordillo, Nelly, Eduard Montseny, and Pilar Sobrevilla. "State of the art survey on MRI brain tumor segmentation." Magnetic resonance imaging 31, no. 8 (2013): 1426-1438.

Kalaiselvi, T., and P. Nagaraja. "Brain tumor segmentation of MRI brain images through FCM clustering and seeded region growing technique." International Journal of Applied Engineering Research 10, no. 76 (2015): 427432.

Kanmani, P., and P. Marikkannu. "MRI brain images classification: a multi-level threshold-based region optimization technique." Journal of medical systems 42, no. 4 (2018): 1-12.

Kansal, Shweta, and Pradeep Jain. ”Automatic seed selection algorithm for image segmentation using region growing." International Journal of Advances in Engineering \& Technology 8, no. 3 (2015): 362.

Lê, Matthieu, Jan Unkelbach, Nicholas Ayache, and Hervé Delingette. "Sampling image segmentations for uncertainty quantification." Medical image analysis 34 (2016): 42-51.

Liu, Jin, Min Li, Jianxin Wang, Fangxiang Wu, Tianming Liu, and Yi Pan. ”A survey of MRI-based brain tumor segmentation methods." Tsinghua science and technology 19, no. 6 (2014): 578-595.

Mabray, Marc C., Ramon F. Barajas Jr, and Soonmee Cha. "Modern brain tumor imaging." Brain tumor research and treatment 3, no. 1 (2015): 8. 
Mohan, Geethu, and M. Monica Subashini. "MRI based medical image analysis: Survey on brain tumor grade classification." Biomedical Signal Processing and Control 39 (2018): 139-161.

Pereira, Sérgio, Victor Alves, and Carlos A. Silva. "Adaptive feature recombination and recalibration for semantic segmentation: application to brain tumor segmentation in MRI." In International Conference on Medical Image Computing and Computer-Assisted Intervention, pp. 706-714. Springer, Cham, 2018.

Shimodaira, Hisashi. "Automatic color image segmentation using a square elemental region-based seeded region growing and merging method." arXiv preprint arXiv:1711.09352 (2017).
Vallabhaneni, Ramesh Babu, and V. Rajesh. "Brain tumour detection using mean shift clustering and GLCM features with edge adaptive total variation denoising technique." Alexandria engineering journal 57, no. 4 (2018): 2387-2392.

Van den Bent, Martin J., Michael Weller, Patrick Y. Wen, Johan M. Kros, Ken Aldape, and Susan Chang. "A clinical perspective on the 2016 WHO brain tumor classification and routine molecular diagnostics." Neuro-oncology 19, no. 5 (2017): 614-624.

$\mathrm{Xu}$, Guangzhu, Xinyu Li, Bangjun Lei, and Ke Lv. "Unsupervised color image segmentation with coloralone feature using region growing pulse coupled neural network." Neurocomputing 306 (2018): 1-16. 